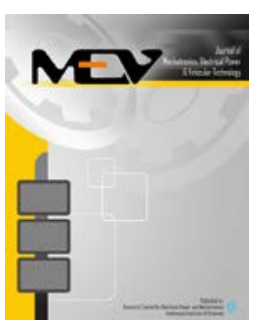

Journal of Mechatronics, Electrical Power, and Vehicular Technology

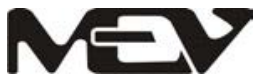

\title{
HARDWARE SimUlation OF AUTOMATIC BRAKING SYSTEM BASED ON FUZZY LOGIC CONTROL
}

\author{
Noor Cholis Basjaruddin ${ }^{a, *}$, Kuspriyanto ${ }^{b}$, Suhendar ${ }^{a}$, Didin Saefudin ${ }^{\text {a }}$, \\ Virna Apriani Azis ${ }^{a}$ \\ ${ }^{a}$ Department of Electrical Engineering, Politeknik Negeri Bandung \\ Jl. Gegerkalong Hilir, Ds.Ciwaruga, Bandung, Indonesia \\ ${ }^{b}$ School of Electrical Engineering and Informatics, Institut Teknologi Bandung \\ Jl. Ganesha no. 10, Bandung, Indonesia
}

Received 04 October 2015; received in revised form 07 December 2015; accepted 14 December 2015 Published online 29 July 2016

\begin{abstract}
In certain situations, a moving or stationary object can be a barrier for a vehicle. People and vehicles crossing could potentially get hit by a vehicle. Objects around roads as sidewalks, road separator, power poles, and railroad gates are also a potential source of danger when the driver is inattentive in driving the vehicle. A device that can help the driver to brake automatically is known as Automatic Braking System (ABS). ABS is a part of the Advanced Driver Assistance Systems (ADAS), which is a device designed to assist the driver in driving the process. This device was developed to reduce human error that is a major cause of traffic accidents. This paper presents the design of ABS based on fuzzy logic which is simulated in hardware by using a remote control car. The inputs of fuzzy logic are the speed and distance of the object in front of the vehicle, while the output of fuzzy logic is the intensity of braking. The test results on the three variations of speed: slow-speed, medium-speed, and high-speed shows that the design of ABS can work according to design.
\end{abstract}

Keywords: automatic braking system; advanced driver assistance system; fuzzy logic.

\section{INTRODUCTION}

Every two hours, pedestrians were killed or every seven minutes, the pedestrian was injured due to being struck by a vehicle in the United States [1]. The insufficiency of braking time owned by the driver is the main reason the vehicle hit a pedestrian. The sudden appearance of pedestrians in front of vehicles often does not allow the driver to brake.

The percentage of vehicles crashing object is around $14.2 \%$ of all types of collisions. The figure has not been merged with rear-end collisions which are caused by the sudden brake or stop of the vehicle in front. About $28 \%$ of the entire collision is a rear-end collision and about $25 \%$ of the collision is due to the insufficiency of the vehicle to brake [2]. Three main factors causing road crashes are the driver, the vehicle, and the infrastructure. In Indonesia, collisions

\footnotetext{
* Corresponding Author.Tel: +62-22-2013789
}

E-mail: noorcholis@polban.ac.id caused by driver error are 93\% [3]. Efforts are being made to reduce the number of traffic accidents involving fixing the vehicles and improve infrastructure technologies as well as to increase the awareness and expertise of drivers.

Automatic Braking System was developed to help drivers cope with braking delay so that the vehicle can avoid the collision. This device is also known as Automatic/Advanced Emergency Braking Systems (AEBS) [4]. ABS is an element of the Advanced Driver Assistance System (ADAS) or Intelligent Driver Assistance System (IDAS). Other elements of ADAS which are Adaptive Cruise Control (ACC) [5], Overtaking Assistance System (OAS) [6], and Lane Keeping Assist System (LKAS) [7].

Honda has used a Collision Mitigation Braking System (CMBS) on products namely Legend Saloon (2006) and CR-V 4x4 (2007). Nissan uses Intelligent Brake System while Mercedes-Benz has equipped the S-Class products (2006) with Brake Assist Plus (BAS 


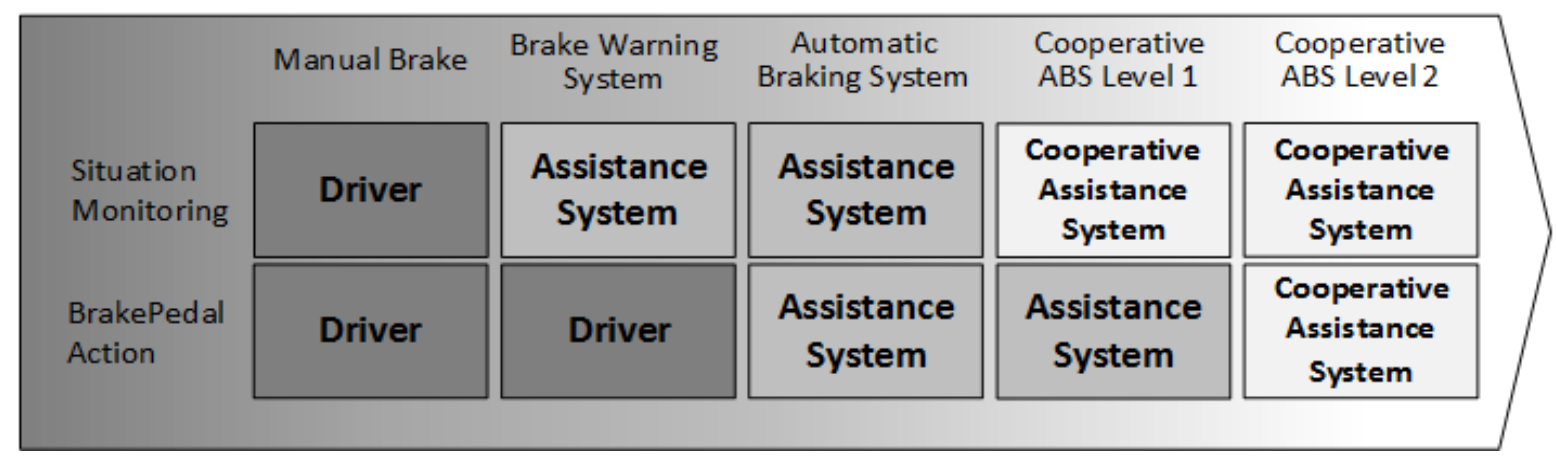

Figure 1. Transition between different levels of ABS

Plus). Ford and Volvo jointly develop the Collision Mitigation by Braking (CMbB). Volvo S80 (2007) and Ford S-Max have been equipped with a Collision Warning with Brake Support System.

ABS technology development can be seen in Figure 1. Braking is done by two-stages that is monitoring the situation and step on the brake pedal. Brake Warning System (BWS) assist the driver in recognizing situations needed for braking. At the time of dangerous situations, BWS will give a warning to the driver for braking. Warnings can be done through sound, light, or haptic [8]. In vehicular networks, the BWS is developed into the cooperative BWS [9]. In certain circumstances such as the existence of a pedestrian crossing the road suddenly, ABS will perform automatic braking. At [10] examined the influence of ABS braking time at the driver's behavior.

Some ABS is developed by combining the role of the driver and braking assistance system in action. At the far distance with obstacle objects, the system will serve as BWS. If the warning is not considered by the driver and the object are near, and then the ABS will perform braking gradually from soft to hard brake. Some research in the development of ABS, among others, performed by [11, 12, 13]. In [11] and [12] were examined the use of cameras for ABS, whereas [13] developed ABS for smart vehicles.

In the cooperative system, the ABS on different vehicles works together through communication systems such as Vehicular Ad hoc NETwork (VANET). Some VANET research conducted were $[14,15]$. Cooperative ABS (CABS) allows the braking a vehicle because some vehicles are far in front of him stopped. Vehicle braking information obtained through inter-vehicle communication such as VANET. CABS enables the braking process can be done early and prevent pileup. Other than that this technology can be developed into Advanced
Adaptive Cruise Control (AACC). In Cooperative ACC situation monitoring and brake pedal action are carried out cooperatively with the aim to improve safety and comfort. Information about when and how the intensity of braking is done by observing the data situation around the vehicle received by the sensor and communication system between vehicles.

\section{Research Method}

Braking is the most important action in the process of driving. Two factors related to the safety braking are when and how the intensity of braking should be done. One of the factors that related to the driving comfort is the magnitude of deceleration when braking process. Braking suddenly would lead to inconvenience the driver as well as passengers. In this study, the driving comfort has not considered due to the difficulties in the remote control car brakes.

\section{A. How ABS work}

Illustration of the workings of the ABS can be seen in Figure 2. ABS will prevent the following vehicle (FV) from a collision with leading vehicle (LV) if LV braking or sudden stop. Collision avoidance process is done by the brakes at braking distance (bd) so that FV car stopped just behind the LV without crashing (stopping distance, sd). Before the ABS gave orders automatic braking, the driver of FV will know that LV decelerates abruptly at a distance of warning distance (wd). If the warning is not taken by the driver with braking, the vehicle is automatically braked at a distance of bd.

Braking at such distances would cause the vehicle to stop at a distance that is still safe (od). Parameters of the system can be seen in Table 1 .

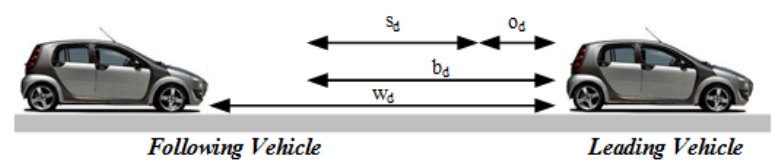

Figure 2. How ABS work 
Table 1.

System parameters

\begin{tabular}{ll}
\hline Parameter & Description \\
\hline bd & braking distance \\
\hline sd & stopping distance \\
\hline wd & warning distance \\
\hline od & obstacle distance \\
\hline $\mathrm{v}$ & The speed of the car \\
\hline br & brake intensity of car \\
\hline
\end{tabular}

Safe braking must meet the conditions bd $>$ sd. The stopping distance (sd) value depends on the vehicle speed and the braking intensity. At highspeed and close distance there is needed of hard braking. In contrast, the low-speed and far distance only needed a soft braking. The main issue on ABS is how to regulate the intensity of the brake (br) so the car will not hit any object in various speed (v) and (bd).

This issue can be resolved by using the on-off method as has been done by [16]. The use of onoff method has drawbacks when applied to the real car is braking intensity are less smooth, thereby reducing driving comfort. This paper

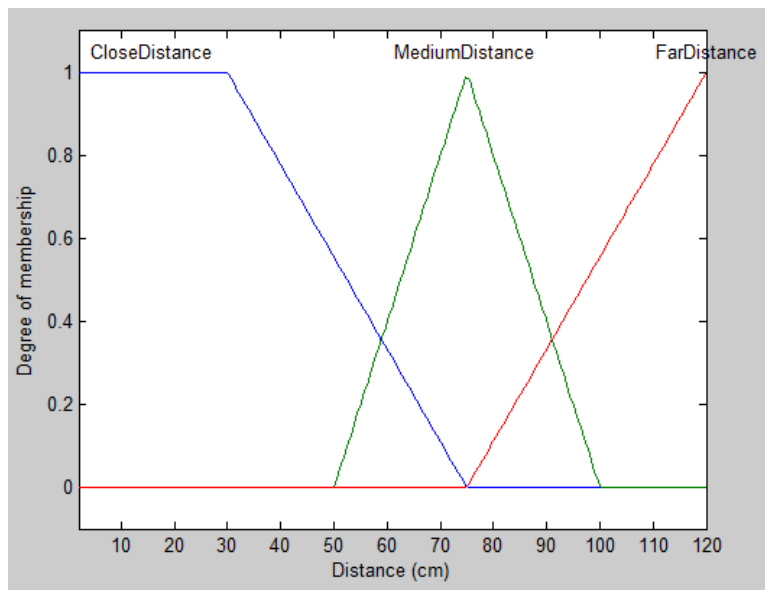

Figure 3. Membership function of distance

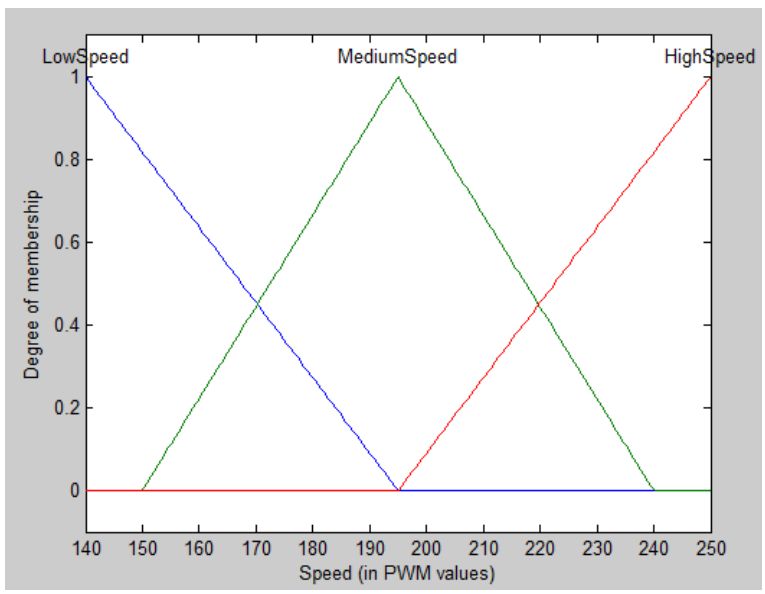

Figure 4. Membership function of speed contains improvement using the on-off method on ABS by using the fuzzy logic method. The use of fuzzy logic is expected to improve the comfort of the real car. Some research has been done in the development of fuzzy logic based ABS among others on $[17,18,19]$.

\section{B. ABS based on Fuzzy Logic}

Input membership functions at the ABS can be seen in Figure 3 and Figure 4 . The membership function of the input consists of the speed and distance of the object in front of the vehicle. Both the membership function is divided into three fuzzy sets are low-speed (LS), medium-speed (MS), and high-speed (HS) for speed and close-distance (CD), medium-distance (MD), and far-distance (FD) for the distance.

Figure 5 shows the output membership function while Figure 6 shows a relationship between input and output on the basis of rules that are shown in Table 2. Membership function output is the braking intensity which is divided into five fuzzy set that is very-soft-brake (VSB), soft-brake (SB), normal-brake (NB), hard-brake (HB), and very-hard-brake (VHB).

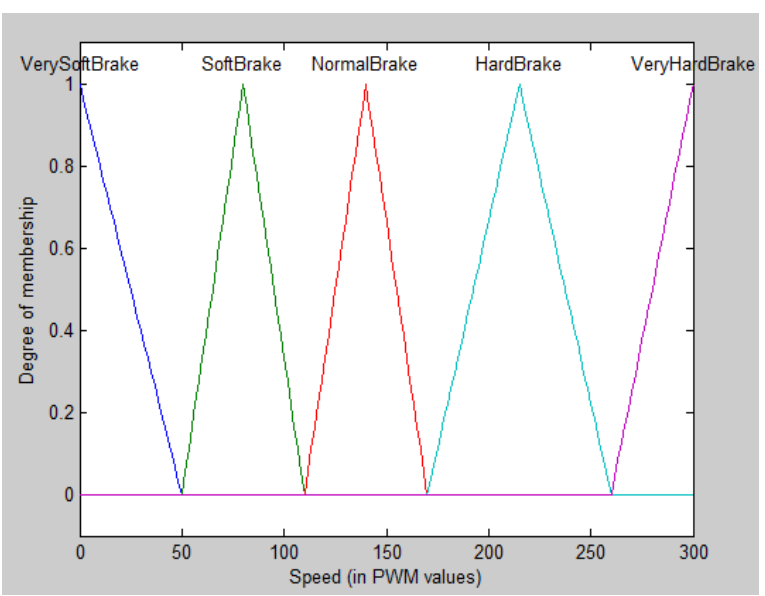

Figure 5. Membership function of output

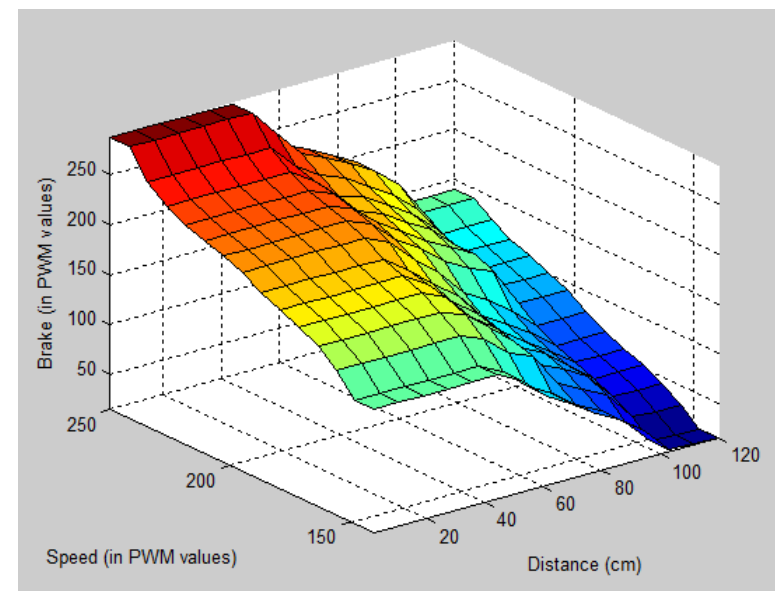

Figure 6. The relationship between input and output 
Table 2.

Rules base

\begin{tabular}{lllll}
\hline & & \multicolumn{3}{c}{ Speed } \\
\hline \multirow{3}{*}{ Distance } & & LS & MS & HS \\
\cline { 2 - 5 } & CD & NB & HB & VHB \\
\cline { 2 - 5 } & MD & SB & NB & HB \\
\cline { 2 - 5 } & FD & VSB & SB & NB \\
\hline
\end{tabular}

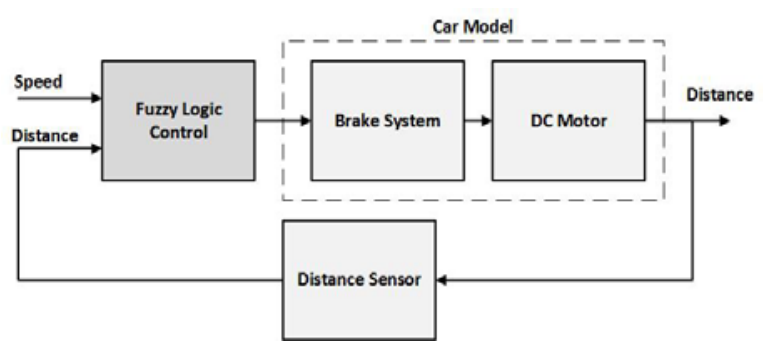

Figure 7. Block diagram of ABS hardware simulation

\section{Hardware Simulation}

The block diagram of the ABS hardware simulation is shown in Figure 7. ABS hardware simulation is built using a remote control (RC) car as shown in Figure 8 and Figure 9 [20]. Hardware simulation used two ultrasonic sensors are which mounted on the front of the car. The sensor is used to measure the distance from LV to $\mathrm{FV}$. Information about object distance is provided by ultrasonic sensor 1 and 2 . The potentiometer is used to adjust the speed of the remote control car. Two Arduino Uno microcontrollers are used in this experiment. One microcontroller is used to run the fuzzy logic based ABS algorithm and perform various other functions such as sensor readings and processing the signal to the actuator. The other microcontroller is used for data logging during the testing process. Measurement results of testing process stored on the SD card.

The Arduino Uno R3 is a microcontroller board based on ATmega328 and library functions for fuzzy logic-based control can be added on the sources code. The library functions are using Min-Max Mamdani method with the center of the area for the defuzzification process as inference method. The important data that obtained during testing procces using hardware simulator is stored in a Secure Digital (SD) memory card via Arduino shield.

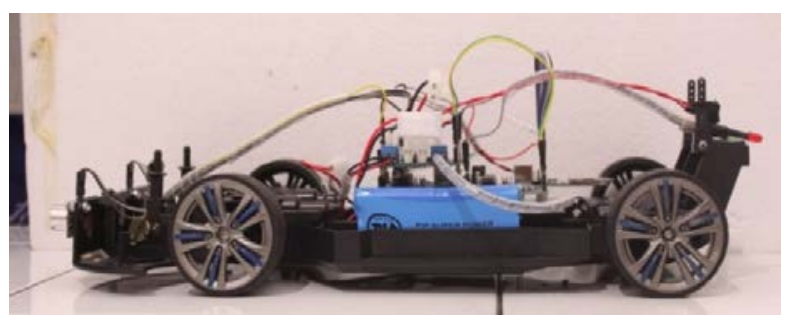

Figure 8. Side view of experiment car

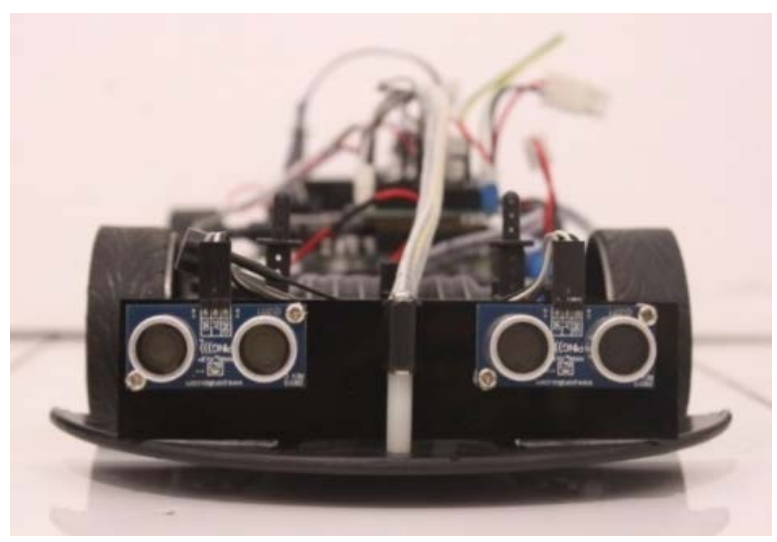

Figure 9. Front view of experiment car

\section{RESULTS AND ANALYSIS}

Fuzzy-logic based ABS algorithm is tested in three situations, namely low-speed, mediumspeed, and high-speed. The distance from an obstacle is assumed to be fixed at $250 \mathrm{~cm}$ and there were observation to the the intensity of the braking so that vehicles do not hit the object, see Figure 10.

\section{A. Testing at Different Speeds}

At each test, the data of speed and distance stored in the SD card. Testing also generates the data of stopping distance, braking distance, and obstacle distance for three different speeds. The relationship between the value of the PWM and the speed of the RC car can be seen in Table 3 .

1) Low-Speed Testing

The results of testing at low-speeds can be seen in Figure 11. The ABS starts working when the distance of the RC car and the obstacle is smaller than $120 \mathrm{~cm}$. Braking is done in accordance with the fuzzy logic control of verysoft-brake, soft-brake, and normal-brake. Car stops when the distance of $32 \mathrm{~cm}$.

Table 3.

PWM value and speed relation

\begin{tabular}{ccl}
\hline PWM & Speed $(\mathbf{m} / \mathbf{s})$ & Speed level \\
\hline 140 & 0.62 & Low-speed \\
\hline 199 & 0.84 & Medium-speed \\
\hline 255 & 1.42 & High-speed \\
\hline
\end{tabular}

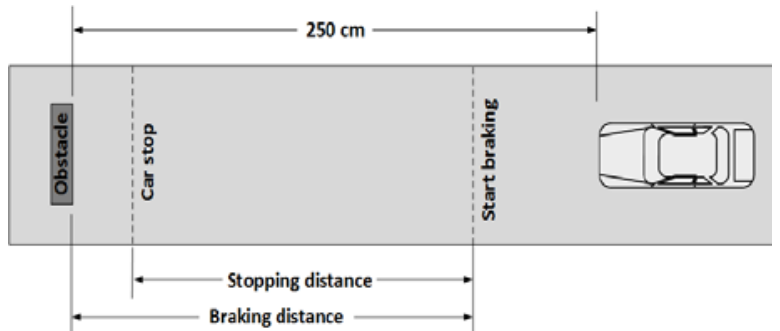

Figure 10. Test scenario 


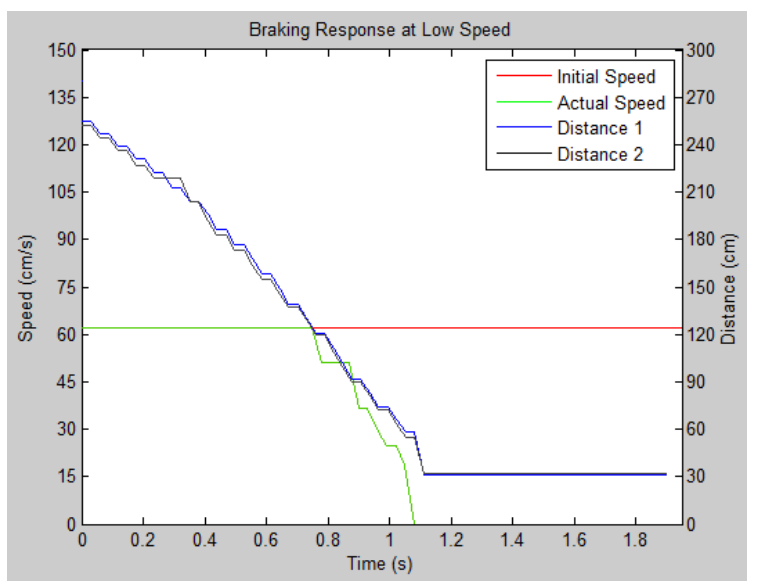

Figure 11. Test results on low-speed

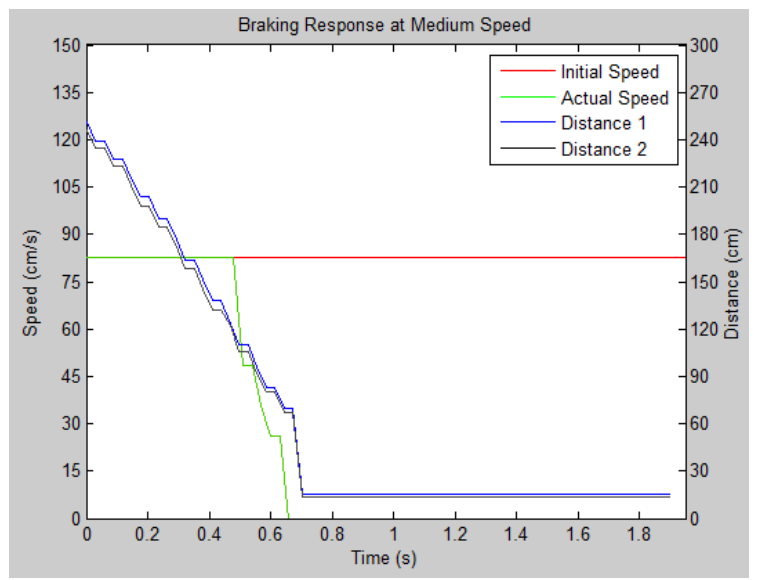

Figure 12. Test results on medium-speed

\section{2) Medium-Speed Testing}

Testing with medium-speed braking is done in accordance with the fuzzy logic control of softbrake, normal-brake, and hard-brake. The car stops when the distance of the RC car and obstacle is $14 \mathrm{~cm}$. The test results in this situation can be seen in Figure 12.

\section{3) High-Speed Testing}

The results of testing at high speeds can be seen in Figure 13. The braking is done in accordance with the fuzzy logic control of normal-brake, hard-brake, and very-hard-brake. The speed of the RC car to zero when the distance of RC car and the obstacle is $5 \mathrm{~cm}$.

\section{B. System Analysis}

The test results for three levels speed can be seen in Table 4. Based on the data obtained on Table 2 it can be seen that when the remote control car speed is greater, then the stopping distance is also greater, and the distance to the obstacle becomes smaller. The braking action begins when the distance is equal or smaller than $120 \mathrm{~cm}$ in accordance with membership function of distance.

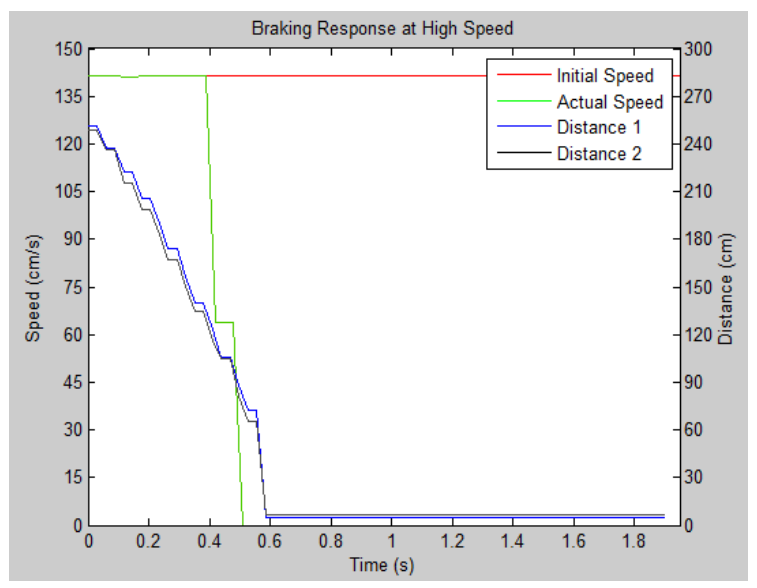

Figure 13. Test results on high-speed

Table 4.

Braking response

\begin{tabular}{lrrr}
\hline Speed Level & $\begin{array}{r}\text { Stopping } \\
\text { distance } \\
\text { (cm) }\end{array}$ & $\begin{array}{r}\text { Braking } \\
\text { distance } \\
(\mathbf{c m})\end{array}$ & $\begin{array}{r}\text { Obstacle } \\
\text { distance } \\
(\mathbf{c m})\end{array}$ \\
\hline Low-speed & 88 & 120 & 32 \\
\hline Medium-speed & 92 & 106 & 14 \\
\hline High-speed & 111 & 116 & 5 \\
\hline
\end{tabular}

The action of braking is done in accordance with the capabilities of the sensor in reading the distance. At low-speed, braking action is done more often than at high-speed. At high-speed, the sensor is often too late in the process of reading distance.

\section{CONCLUSION}

The results show that the hardware simulated of ABS can work well. The fuzzy-logic based ABS algorithm can set when and how the intensity of braking is needed so that the car does not crash into an obstacle. At high-speed (1.42 $\mathrm{m} / \mathrm{s}$ ), the sensors are too late to measure the distances thereby affecting to ABS work.

\section{ACKNOWLEDGEMENT}

The authors would like to thank Unit Penelitian dan Pengabdian Masyarakat (UPPM) Politeknik Negeri Bandung and Mechatronic Laboratory at Electrical Departement, Politeknik Negeri Bandung for supporting in this research. Authors wish to thank the DIKTI DP2M for financial support through Penelitian Hibah Bersaing 2014 and 2015.

\section{REFERENCES}

[1] NHTSA, "Traffic Safet Facts," National Center for Statistics and Analysis, NHTSA, Washington, DC, 2014. 
[2] Henrik Clasen, "Rear-End Accident Statistics Stationary Vehicles vs. Stopped and Moving," CLEPA, Sweden, 2009.

[3] Sutanto Soehodo, "Road Accidents in Indonesia," IATSS Research, vol. 33, no. 2, 2009.

[4] C Grover et al., "Automated Emergency Brake Systems: Technical requirements, costs and benefits," TRL, 2008.

[5] Noor Cholis Basjaruddin et al., "Developing Adaptive Cruise Control Based on Fuzzy Logic Using Hardware Simulation," International Journal of Electrical and Computer Engineering (IJECE), vol. 4, no. 6, 2014.

[6] Noor Cholis Basjaruddin et al., "Overtaking Assistant System based on Fuzzy Logic," Telkomnika (Telecommunication Computing Electronics and Control), vol. 13, no. 1, 2015.

[7] Noor Cholis Basjaruddin et al., "Lane Keeping Assist System Based on Fuzzy Logic," in 2015 International Electronics Symposium (IES), PENS, Surabaya, 2015.

[8] John L. Campbell et al., "Crash Warning System Interfaces:Human Factors Insights and Lessons Learned," Washington, DC, 2007.

[9] Ming-Fong Tsai et al., "Cooperative emergency braking warning system in vehicular networks," Journal on Wireless Communications and Networking, vol. 2015, 2015.

[10] Takahiro Wada et al., "Effect of Activation Timing of Automatic Braking System on Driver Behaviors," in SICE Annual Conference, Taipei, 2010.

[11] Vicente Milanés et al., "Vision-based active safety system for automatic stopping," Expert Systems with Applications, vol. 39, 2012.
[12] Christoph G. Keller et al., "Active Pedestrian Safety by Automatic Braking and Evasive Steering," IEEE Transaction on Intelligent Transportation Systems, vol. 12, no. 4, December 2011.

[13] Shrey Modi et al., "A Driver-Automation System for Brake Assistance in Intelligent Vehicles," in 10th IEEE International Conference on Industrial Informatics (INDIN), Beijing, 2012, pp. 446 - 451.

[14] G. Chandrasekaran, "VANETs: The Networking Platform for Future Vechicular Applications," in Mobile Networking for Vehicular Environments, Los Angeles, 2009, pp. 109-114.

[15] Sherali Zeadally et al., "Vehicular ad hoc networks (VANETS): status, results, and challenges," Telecommun Syst, 2010.

[16] Wahyu Eko Phasa, "Automatic Braking System on Remote Control Car," Bandung, 2013.

[17] ML Sharma and Sheetal Atri, "Fuzzy Rule based Automatic Braking System in Train using VHDL," International Journal of Computer Science and Technology, vol. 2, no. 2, pp. 332-335, 2011.

[18] J.H Li and H.M Kim, "Design for the Predictor of the Emergency Braking System Based on Fuzzy Algorithm," Journal of Automation and Control Engineering Vol.1 No.3, vol. 1, no. 3, pp. 240-244, 2013.

[19] Dhivya P and Murugesan A, "Intelligent Car Braking System with Collision Avoidance and ABS," International Journal of Computer Applications, 2015.

[20] Virna Apriani Azis, "Automatic Braking System Based on Fuzzy Logic," Electrical Engineering, Politeknik Negeri Bandung, Bandung, Final Project 2014. 